world's most eminent workers in the field of skeletal and cardiac muscle physiology. Half the Symposium is devoted to studies on isolated muscle, with special reference to work at microscopic level. Here, there is some exacting work on the structure of skeletal muscle, with discussions on the practical significance of the length-tension curve and the force-velocity relationship.

The second half of the Symposium deals with the intact heart and contains some interesting work involving direct measurement of tension in the intact heart, and ends with an excellent discussion on homeometric autoregulation. Each chapter consists of a paper, followed by a discussion on the subject matter. There are also four general discussions at various stages throughout.

The subject-matter is essentially physiological and, though attractively set out, with useful diagrams and photographs, it demands much concentration in reading. However, despite the complexity of the subject discussed, the symposium does give an insight into recent sophisticated research tools such as the use of laser beams, light and $X$-ray diffraction, and cinemicrography. The discussions at the end of each chapter are good and quite entertaining and informative-their informality being their strength. Here there are many excellent contributions, especially from one of the great experts in this field, E. H. Sonnenblick.

However, this Symposium does present much complex material which is not directly relevant or easily assimilated by the average practising physician today. It will be, though, of great interest to physiologists, and cardiologists with an interest in cardiac muscle physiology. It also suffers from the lack of a formal conclusion. It is marvellously introduced in a stimulating introduction by the Chairman, Dr A. Guz. However, at the end one is rather left in the air grasping back at a great many complex facts and ideas; a formal conclusion, used to draw all the various subjects together as a whole, would have been most beneficial.

\section{The Epilepsies. Modern diagnosis and treatment.}

By J. M. Sutherland, H. Tait and M. J. Eadie. Second edition. Pp. 149, soft cover. Edinburgh: Churchill Livingstone, 1974. $£ 2.50$.

This is an excellent book containing all that is needed for the General Practitioner to know about the diagnosis and treatment of the epilepsies.

It is said that there are almost as many classifications of epilepsy as there are epileptologists and, certainly, the classification of the International League against Epilepsy is far too cumbersome for routine clinical practice. The authors have recognized this and produced a neater approach. The surgical treatment rightly takes only four pages since less than $2 \%$ of patients require this form of treatment.

It is a clear, beautifully produced book and can be thoroughly recommended for anyone concerned with the management of patients with epilepsy.

\section{Micromanipulation of Bacteria}

By K. I. Johnstone. Pp. 110. Edinburgh and London: Churchill Livingstone, 1973. $£ 4.00$.

Technical procedures performed with single bacterial organisms are used for a variety of reasons. The techniques involved are generally very demanding and laborious. Dr Johnstone has devised simplified methods which can be generally applied and which enable single organisms to be manipulated with greater facility than was formerly possible. This book describes the techniques of agar-gel dissection developed by the author over many years of work in this field.

The book is a model of clarity. It is well written and is concise. Within the space of 110 pages, including a large number of admirably clear diagrams, the reader is given every scrap of information necessary to enable him to carry out the procedures described. All this has been achieved without making the book difficult to read. It is agreeable to be able to recommend a book so whole-heartedly. The main readership of this book will probably be among postgraduate microbiologists since the specialized problems covered will be of practical concern to few medical bacteriologists.

Psychiatric Illness. Diagnosis, Management and Treatment for General Practitioners and Students

By H. Merskey and W. L. Tonge. Second edition. Pp. 292. London: Bailliére Tindall, 1974. $£ 2.80$.

I would highly recommend this little book to students and postgraduates alike. It manages to give a balanced, concise and yet comprehensive account of current thinking in psychiatry. The range is remarkable for such a small volume e.g. it includes useful accounts of such varieties of treatmento as behavioural approaches to sexual disorder as well as
standard accounts of the drug treatment of functionat psychoses. It seems very well balanced because sociafe dynamic and organic factors in aetiology are all discussed? Above all, readers will find it very practical in its approach. It is pleasing, too, that there are clear, dogmatic statements concerning drug treatment and no fear of common trade names to simplify the problems.

It is an excellent introduction to the subject and perhaps for the next edition an additional chapter on the role of the social worker and his/her contribution could be included.

\section{Psychogeriatrics. An Introduction to the Psychiatry of Old Age}

By Brice Pitr. Edinburgh and London: Churchill Livingstone, 1974. $£ 2.25$.

This is a most useful and readable account of the subject. The enthusiasm and the optimism which the Author transmits in his writing are most valuable lessons to all. I think it is these attitudes which are most likely to convince that the psychiatry of old age is a discipline in its own right.

Perhaps a chapter on the problems of nursing the elderly in institutions would have been helpful as all too often the nurse is left in an intolerable situation with the technical and emotional problems of the elderly and their relatives.

This is an excellent survey of the whole subject and should be recommended to all postgraduates. 